\title{
Low-Head Energy Conversion: A Conceptual Design and Laboratory Investigation of a Microtubular Hydro Propeller
}

\author{
Helena M. Ramos, Mariana Simão, and Kaloyan N. Kenov \\ Dipartimento di Ingegneria Civile, CEHIDRO, Instituto Superior Técnico, 1049-001 Lisbon, Portugal \\ Correspondence should be addressed to Helena M. Ramos, hr@civil.ist.utl.pt
}

Received 20 October 2011; Accepted 23 November 2011

Academic Editor: S. W. Chang

Copyright (๑) 2012 Helena M. Ramos et al. This is an open access article distributed under the Creative Commons Attribution License, which permits unrestricted use, distribution, and reproduction in any medium, provided the original work is properly cited.

Low-head microhydro systems for energy production are becoming accepted because of oil crises and new advances in their design and efficiencies. As this is still a new area for development and experimentation, it is important to test and validate the optimal conditions and the hydrodynamic behaviour of such systems under different conditions. The aim of this paper is to present a turbine design validation practise, which researchers and equipment manufactures can use for the hydrodynamic behaviour analysis of new low-head turbines prior to production and application. Laboratory experimental tests and advanced CFD numerical analysis are described for the flow behaviour analysis of a new prototype microtubular propeller. Laboratory experimental results are presented and used for the evaluation of the turbine performance curves. Comparisons between experimental and CFD results are also presented. Finally, an assessment of the hydrodynamic behaviour is made for a scaled model application, using the theory of turbomachine similarity.

\section{Introduction}

Despite that researchers and equipment manufactures have paid less attention to the emerging field of low-head power engines, microturbines can provide innovative, environmental friendly, and cost-effective solutions for energy production. The challenge is to provide new turbine designs, which can be customized and applied to existing water systems, characterized by low-head and nearly constant flow rates.

Microhydropower, generally, refers to power below $5 \mathrm{~kW}$. Blakely and O'Connor [1] and, most recently, Campbell [2] has reported technologies, prospects, and potential uses of micro-hydro-electric schemes in remote locations. Howey [3] built and tested a fixed geometry propeller turbine for a runner speed of $1000 \mathrm{rpm}$, suited up to a $35 \mathrm{~m}$ head, and at about a 5 1/s flow rate, documenting good efficiencies. Simpson and Williams [4] also designed and field-tested a fixed geometry propeller turbine with a spiral casing, showing an overall mechanical efficiency of $65 \%$. Several researchers (Demetriades [5], Upadhyay [6], Alexander et al. [7-10], and, most recently, Skotak et al. [11]) have developed medium-sized models of propellers turbines.
Olgun $[12,13]$ investigated the performance of a crossflow turbine as a source of hydropower generation for small and microsystems, especially for low-head establishments. Sumathy et al. [14] presented analysis of the operation and performance of a solar thermal water pump. Krishnamoorthy et al. [15] reported design optimization studies conducted on a novel steam-operated pistonless pump, fuelled by biomass. Sutikno and Adam [16] presented research on the design, simulation, and experimental work of a very lowhead turbine. Kaniecki and Krzemianowski [17] reported the complete design process for a low-head propeller hydraulic turbine.

Until now, very few to no studies have been conducted for propellers working as microturbines, especially for solutions working under low-power rates particularly designed to be implemented in existing water pipes through a bypass to the system (e.g., in water supply systems), allowing for new developments and improvements in their design, efficiency, size, and stability of operation. The design solutions found in literature are usually appropriate for hydropower schemes with large discharge values. Smaller and optimized blade geometries, applied to low-power flow, especially associated 


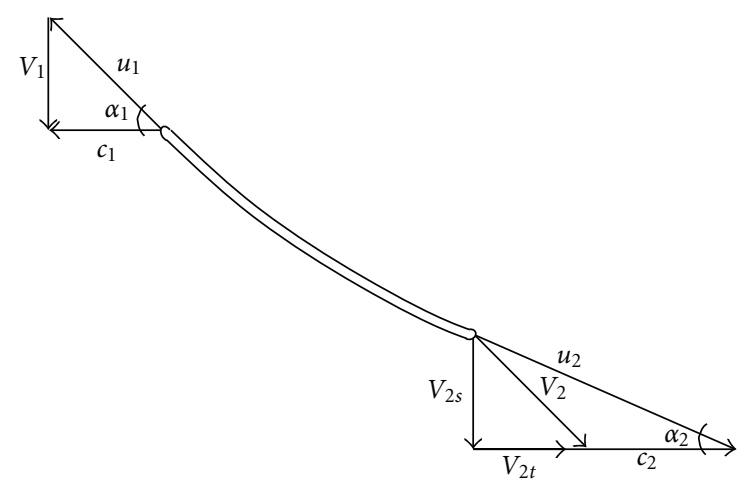

FIGURE 1: Velocity vectors in a blade of a turbine propeller.

with water pipe systems, are still underrepresented in literature and in particular in practise.

According to Ramos et al. [18], the behaviour of such microhydro converters can differ depending on the flow conditions and on the runner configuration, particularly, on the inlet and outlet flow and on the shape of the blades. The design of new low-head devices cannot be solely based on a large turbine scaling-down methodology. To overcome this limitation, CFD analysis can be used to provide design and hydrodynamic behaviour information, which can strongly support the experimental tools. CFD analysis is important to help in the design of the best configuration for different system's characteristics and flow scenarios, according to Rawal and Kshirsagar [19].

This paper aims to close the existing gap found in literature on how to obtain the optimal design of low-power turbines. Focus is put on a methodology and on a new design, which couples together mathematical modelling, CFD numerical analysis, and laboratory testing in order to validate the optimal performance of a new microtubular low-head converter. This is an important topic because water supply and distribution management systems are interested in this solution, and as this new class of low-head converters can be implemented as decentralized solutions in pressurized water pipe infrastructures (e.g., in water supply, irrigation and waste water, or drainage systems) in rural and remote areas for alternative and low-cost energy production.

\section{Turbine Design}

To determine optimal design results, which to lead to the best-efficiency point (BEP) of a prototype turbine, it is important to analyse different blade slopes (i.e., angle variation) when designing the impeller blades. The thickness of the impeller blades should also be observed to avoid disturbances into the flow, which can cause additional losses, and may constrain the overall efficiency. A maximum thickness of $1 \mathrm{~mm}$ was considered in this study, in order to avoid limitations in the CFD mesh created for the model simulations.

Figure 1 shows the velocity triangles associated with the optimization of the blade configuration. It also shows the parameters related to each other from the direction of the blade profiles, such as the angle variation, as indicated by the vectors. A blade model configuration (BMC) was developed to estimate the best blade orientation, which to lead to best efficiency operating conditions, as reported by Ramos et al. [20]. The proper blades orientation is a computationally and experimentally demanding process, which requires attention and performance of sensitivity analysis for different characteristic parameters associated with the inlet and outlet velocity triangles. Figure 1 also identifies the vectors of absolute $(v)$, periphery $(c)$, and relative runner blade velocity $(u)$. Essential relationships are established among them to calculate the turbine discharge for a given configuration. The periphery velocity $(c)$ at the inlet and outlet of a blade, which depends on the impeller rotational speed $(\omega)$ and on the blade radius $(r)$, can be obtained by (1)

$$
c_{1}=c_{2}=\omega r,
$$

and the absolute velocity $(v)$ depends on the discharge $(Q)$ which passes through the impeller, given by (2)

$$
v_{1}=v_{2 s}=\frac{Q}{S}=\frac{Q}{\pi\left(r_{e}^{2}-r_{i}^{2}\right)},
$$

where $S$ is the tubular cross-section area, and $r_{e}$ and $r_{i}$ are the tip and hub blade radius between the runner periphery and internal bulb, respectively.

The blade angles $\left(\alpha_{1}\right.$ and $\left.\alpha_{2}\right)$ on the periphery of the inlet and outlet (subscript 1 and 2, resp.) yield the following (3):

$$
\begin{gathered}
\operatorname{tg} \alpha_{1}=\frac{v_{1}}{c_{1}}=\frac{Q}{S} \cdot \frac{1}{\omega r}, \\
\operatorname{tg} \alpha_{2}=\frac{v_{2 s}}{c_{2}+v_{2 t}}=\frac{v_{1}}{\omega r+v_{2 t}} \Leftrightarrow \operatorname{tg} \alpha_{2}=\frac{Q}{S}\left(\frac{1}{\omega r+v_{2 t}}\right) .
\end{gathered}
$$

Analysis of the flow at downstream of the impeller reveals a vortex formation $(k)$, which depends on the radius of the blade, the flow cross-section and on the discharge value as presented in (4)

$$
v_{2 t}=\frac{k}{r} \Longrightarrow k=-\omega r+\frac{Q r}{\operatorname{Stg} \alpha_{2}} .
$$

As the blade angle changes from the inlet to the outlet, between the upstream turbine section, where the flow impulses the blade, to the downstream turbine section, where 


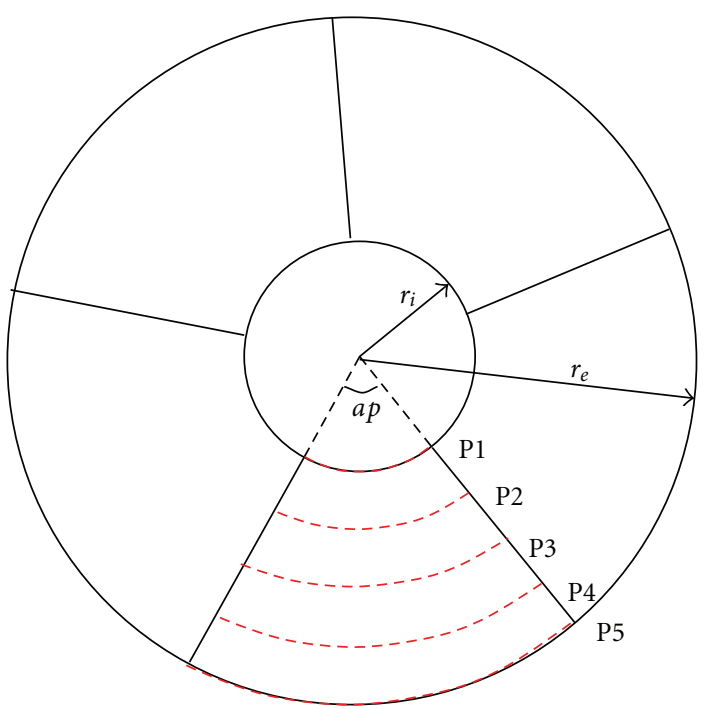

(a)

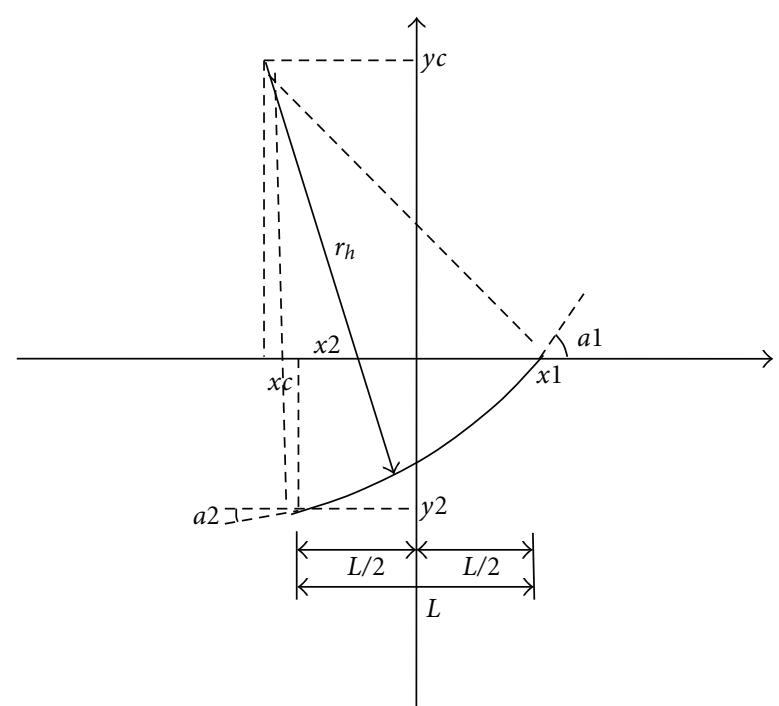

(b)

Figure 2: Scheme of a propeller: (a) plan view and five profiles in a blade and (b) parameters associated to the tracing profiles in each blade.

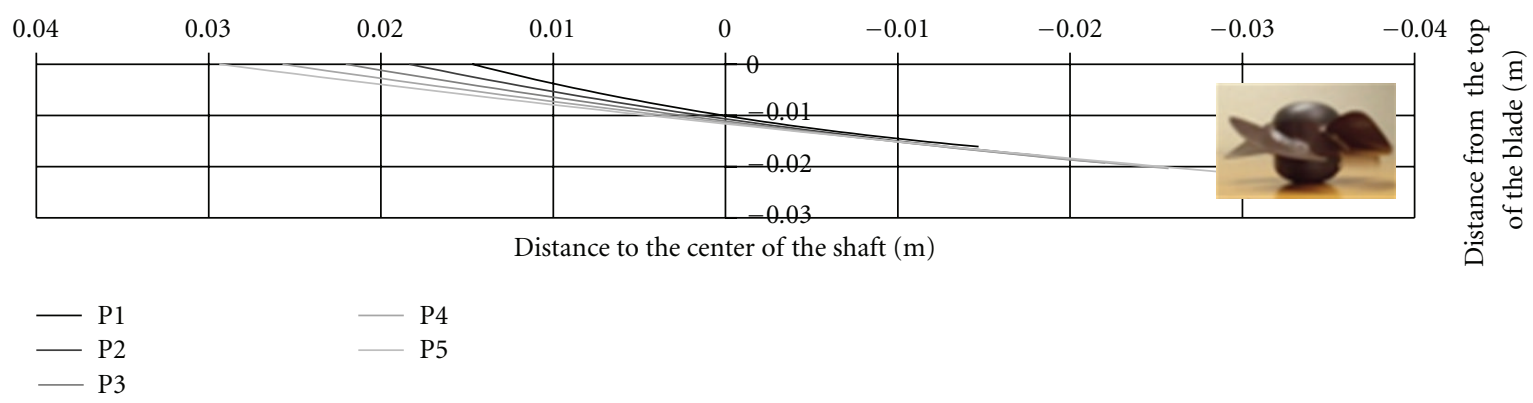

FIGURE 3: Design of different profiles for each blade. Impeller configuration.

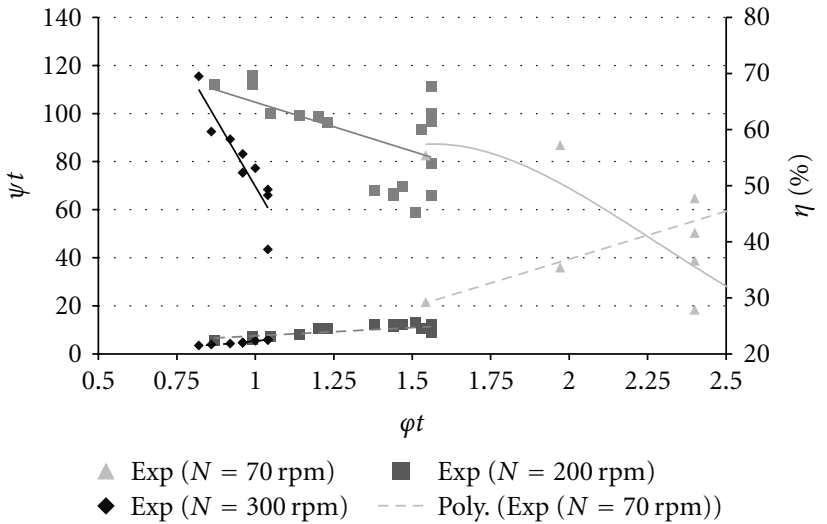

FIgURE 4: Characteristics curves of the micro-tubular propeller.

the flow exits the machine, the overall efficiency changes. This may lead to better performance, depending on the angular variation along the blade profile. Based on (3) to (4), the $\mathrm{BMC}$ establishes the slope blade variation for a given rotational speed, leading to an optimum performance.
Using (4) and values for head, discharge, rotational speed, and runner diameter, the relationship between the runner bulb and periphery diameter, the open blade angle ( $a p$ ) (see Figure 2), which depends on the number of blades, the angles of the inlet and outlet from the axis to the periphery in each blade, can be calculated. It is also possible to calculate the power, the specific speed, and the constant vortex velocity. Having data from the input conditions, the correct blade configuration from the bulb section to the periphery (Figure 3 ) is then determined.

Torque $(M)$, impeller rotational speed, fluid mass density $(\rho)$, discharge, and free-vortex constant are used to calculate the engine or mechanical power $\left(P_{\mathrm{mec}}\right)$, by using (5),

$$
P_{\mathrm{mec}}=M \omega=\rho Q k \omega,
$$

the hydraulic power $\left(P_{h}\right)$ is obtained by using the specific weight of the fluid $(\gamma)$, the discharge, and the net head $(H)$ in (6),

$$
P_{h}=\gamma Q H,
$$


TABLE 1: Values for the blade profile configuration.

\begin{tabular}{|c|c|c|c|c|c|c|c|c|c|}
\hline & $r(\mathrm{~m})$ & $\operatorname{tg}(a 1)$ & $\operatorname{tg}(a 2)$ & $L(\mathrm{~m})$ & $r_{h}(\mathrm{~m})$ & $x_{1}(\mathrm{~m})$ & $x_{2}(\mathrm{~m})$ & $x_{c}(\mathrm{~m})$ & $y_{c}(\mathrm{~m})$ \\
\hline \multirow{2}{*}{ P1 } & 0.0250 & 0.8646 & 0.2975 & 0.0354 & 0.0958 & 0.0177 & -0.0177 & -0.0450 & 0.0725 \\
\hline & 0.0281 & 0.7685 & 0.3066 & 0.0398 & 0.1258 & 0.0199 & -0.0199 & -0.0568 & 0.0997 \\
\hline \multirow{2}{*}{ P2 } & 0.0313 & 0.6917 & 0.3116 & 0.0442 & 0.1628 & 0.0221 & -0.0221 & -0.0705 & 0.1339 \\
\hline & 0.0344 & 0.6288 & 0.3131 & 0.0486 & 0.2082 & 0.0243 & -0.0243 & -0.0865 & 0.1762 \\
\hline \multirow{2}{*}{ P3 } & 0.0375 & 0.5764 & 0.3120 & 0.0530 & 0.2632 & 0.0265 & -0.0265 & -0.1049 & 0.2280 \\
\hline & 0.0406 & 0.5321 & 0.3090 & 0.0575 & 0.3292 & 0.0287 & -0.0287 & -0.1259 & 0.2907 \\
\hline \multirow{2}{*}{ P4 } & 0.0438 & 0.4941 & 0.3045 & 0.0619 & 0.4080 & 0.0309 & -0.0309 & -0.1498 & 0.3658 \\
\hline & 0.0469 & 0.4611 & 0.2990 & 0.0663 & 0.5011 & 0.0331 & -0.0331 & -0.1767 & 0.4551 \\
\hline P5 & 0.0500 & 0.4323 & 0.2928 & 0.0707 & 0.6104 & 0.0354 & -0.0354 & -0.2069 & 0.5603 \\
\hline
\end{tabular}
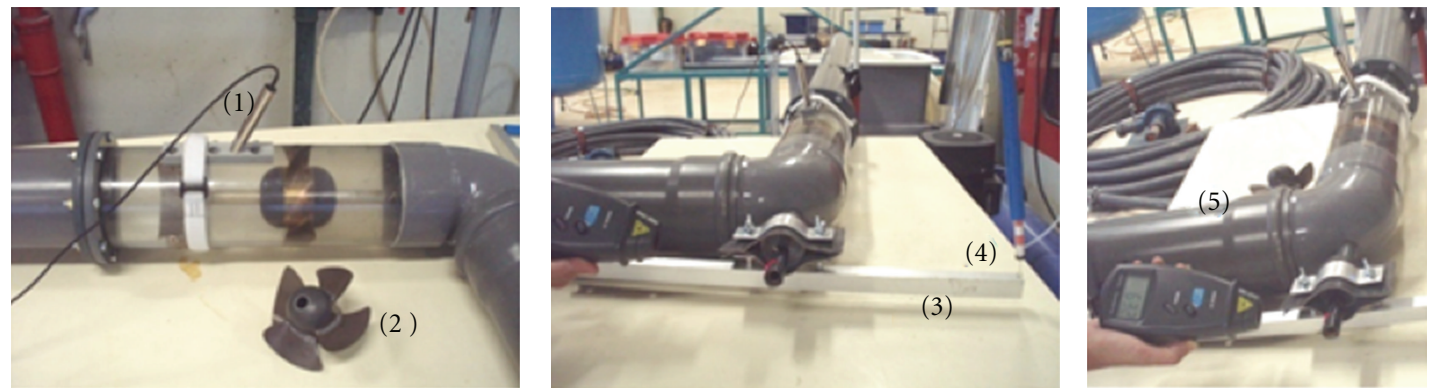
(1) UDV
(2) Propeller
(4) Dynamometer
(3) Balance torque
(5) Tachometer Hibok-24

FIGURE 5: Experimental facility of the micro-tubular propeller: UDV (left), balance torque (center), and rotational speed measurement (right).

and the efficiency $\left(\eta_{t}\right)$ can be obtained by (7), using the mechanical and the hydraulic power

$$
\eta_{t}=\frac{P_{\mathrm{mec}}}{P_{h}}=\frac{\rho Q k \omega}{\gamma Q H}=\frac{k \omega}{g H} .
$$

Outer impeller diameter of $100 \mathrm{~mm}$, with a bulb diameter of $50 \mathrm{~mm}$, is considered for the design of this small-scale tubular propeller. This propeller design is adequate for laboratory testing and for the most of real water distribution system conditions with small flow discharge. According to Table 1, the blades design is built to operate with a discharge around $5 \mathrm{l} / \mathrm{s}$ and at a maximum rotational speed of $300 \mathrm{rpm}$. To design the blades profile, presented in Figure 3, the variables presented in Table 1 are used in (3) to (6).

\section{Experimental Tests}

In order to maintain a continuous steady-state flow conditions, an experimental loop pipe system was developed for the analysis of the flow through a prototype of a micropropeller turbine with the impeller diameter of $D=$ $100 \mathrm{~mm}$. This setup consists of a pressure pipe system with an installed pump for recirculation, an air vessel for upstream pressure control, an electromagnetic flow meter, and a downstream reservoir with a triangular $\left(90^{\circ}\right)$ weir. A valve is used for flow control. When the valve is fully open, the maximum possible turbine flow is $5.2 \mathrm{l} / \mathrm{s}$.

In order to estimate the output power, the shaft transmits the momentum to a torque balance through the turbine upstream curve. During testing, isotropic behaviour of the flow upstream of the turbine was observed. Observed were also few anisotropic occurrences through the impeller, influenced by the flow rotation and separation flow zone of the boundary layer, which exists downstream of the internal impeller bulb (as presented in Figure 6).

Under laboratory conditions, the best efficiency point (BEP) for the micro-tubular propeller $(D=100 \mathrm{~mm})$ was estimated at about $70 \%$ for a rotational speed of $200 \mathrm{rpm}$ $\left(N_{\text {sqt }}\right.$ (specific rotational speed $)=84 \mathrm{rpm}\left(\mathrm{m}, \mathrm{m}^{3} / \mathrm{s}\right)$ based on (10)), as shown in Figure 4. The performance dimensionless curves are based on efficiency (from (7)), the head number, and discharge number, defined as

Discharge number $(\varphi)$ :

$$
\varphi=\frac{Q}{N D^{3}}
$$

Head number $(\Psi)$ :

$$
\psi=\frac{g H}{N^{2} D^{2}},
$$

where $N$ is the rotational speed, and $D$ is the pipe diameter or outside diameter of the blades. 


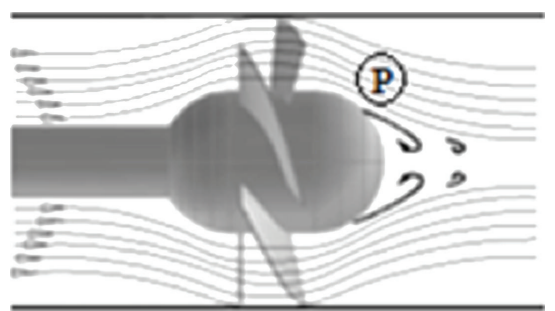

(a)

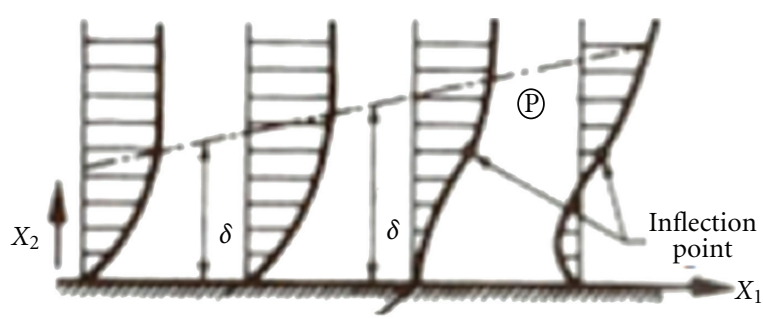

$\left(\frac{\partial v_{1}}{\partial x_{2}}\right)_{0}>0\left(\frac{\partial v_{1}}{\partial x_{2}}\right)_{0}=0\left(\frac{\partial v_{1}}{\partial x_{2}}\right)_{0}<0$

(b)

FIGURE 6: Separation of the boundary layer and velocity profiles.

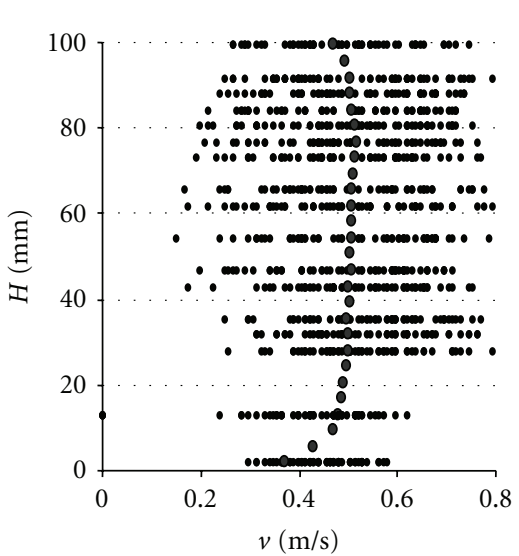

(a)

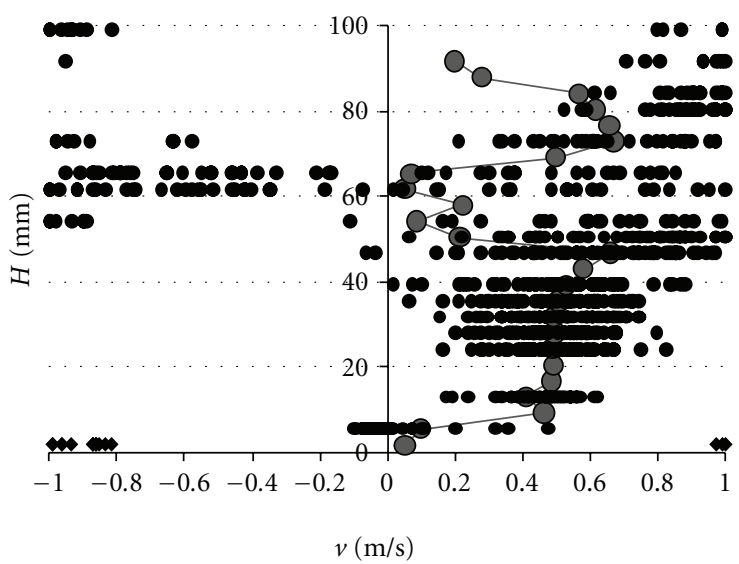

(b)

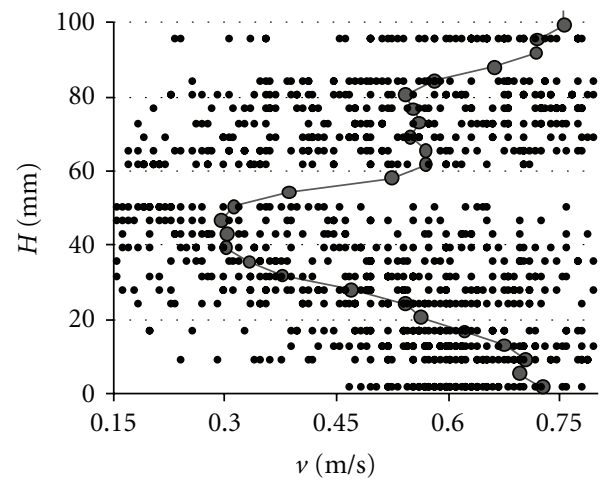

(c)

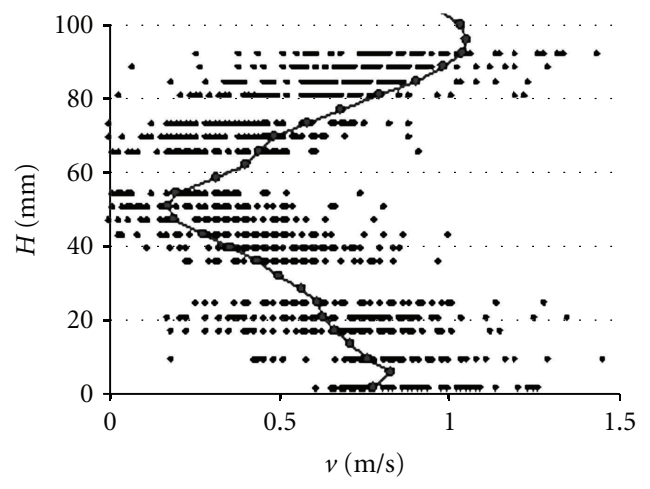

(d)

FIGURE 7: Flow velocity profiles obtained by UDV along the turbine: (a) at upstream in the nonpropeller disturbed zone, (b) at the upstream shaft, (c) in the middle of the impeller, (d) at downstream of the impeller.

Under laboratory conditions, experimental results are obtained by regulating the discharge control valve, measuring the runner speed, with a tachometer Hibok-24 for different flow values, the discharge and head, in an electromagnetic flow meter and in pressure transducers at upstream and downstream of the turbine, in undisturbed flow zones. Using an ultrasonic Doppler velocimetry (UDV) in the turbine system (Figure 5), the velocity profiles were analysed. With the UDV sample placed in a vertical-sloped position of $25^{\circ}$, this device was used for the evaluation of the flow hydrodynamic behaviour in real-time situation. Figures 6 and 7 show different velocity profiles along the runner boundary layer, where the behaviour of the flow separation zone is represented, and in which the velocity profile inversion tendency is visible. The most important features during the identification of instantaneous flow velocity values and mean profiles (Figure 7) are due to: (a) flow irregularity which is indicated by the occurrence of three-dimensional vorticity 


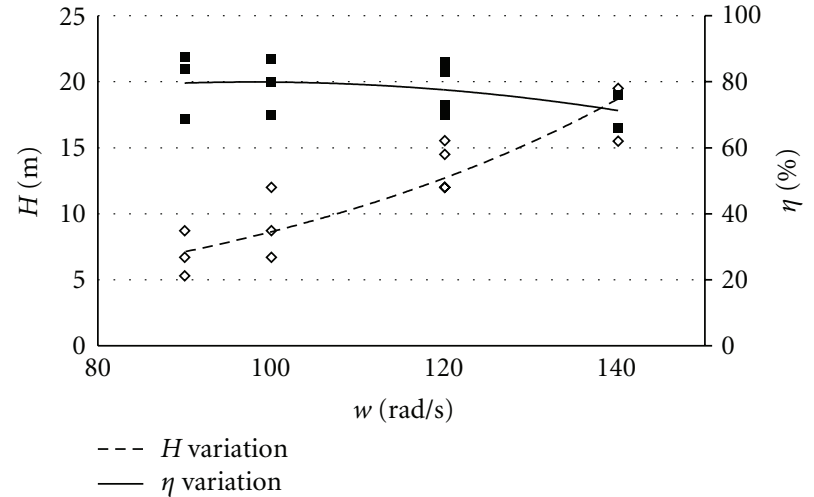

(a)

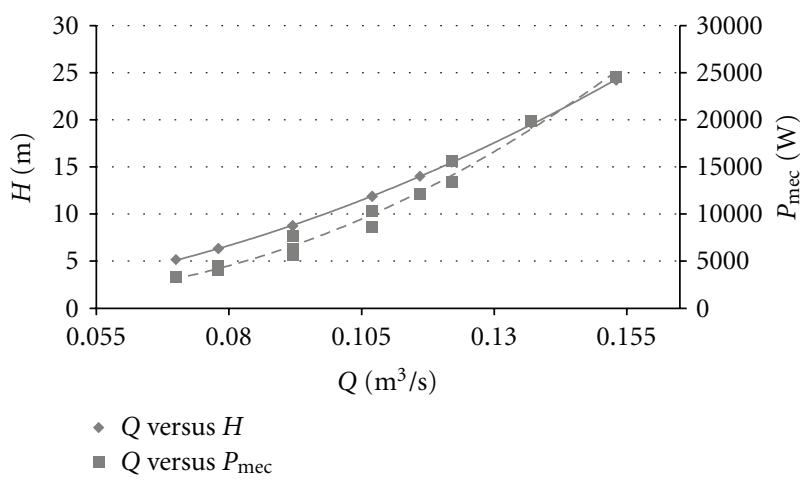

(b)

FIGURE 8: Performance curves for a runner diameter of $200 \mathrm{~mm}$ : (a) efficiency and head versus rotational speed and (b) head and mechanical power versus discharge.

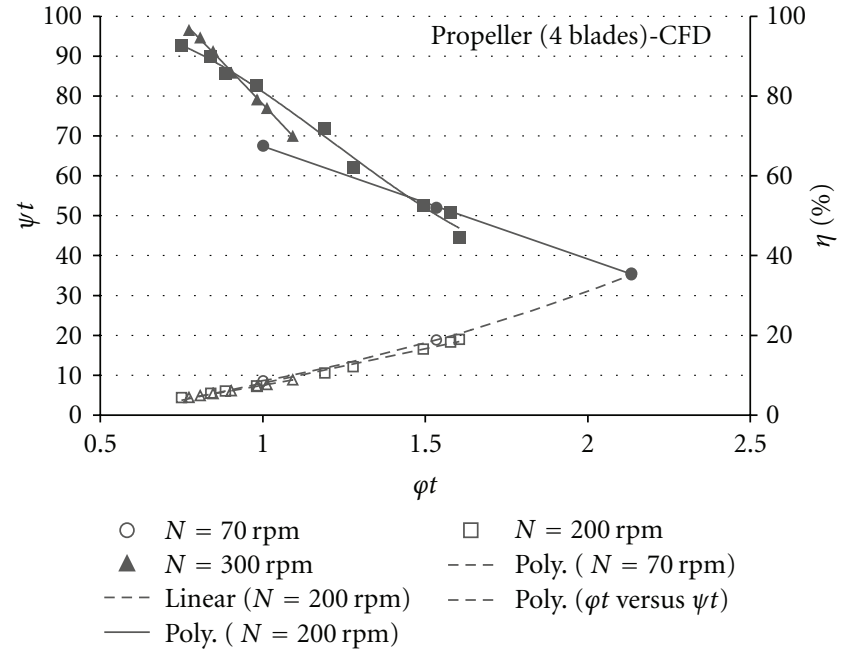

Figure 9: Comparison of power and head numbers and efficiency versus discharge number for different rotational speeds with $D=$ $100 \mathrm{~mm}$.

fluctuations, that is, the turbulent movements are of the rotational type, (b) energy dissipation, that is, the turbulent phenomenon is associated with a significant energy loss, where the turbulence is damped quickly by giving a greater homogeneity and isotropy to the flow motion, (c) diffusivity, corresponding to a rapid mixing within the fluid domain, followed by transfer of momentum, heat and mass, in rapid variations or fluctuations in the flow.

\section{Numerical Modelling}

4.1. Performance Curves. Comparisons between BMC and 3D-CFD simulations were developed for an impeller design with four blades, characterised by the specific speed, as stated in $(10)$

$$
N_{s q t}=N \frac{\sqrt{Q}}{H^{0.75}} .
$$

From Table 2 it is possible to confirm the good match between the BMC (maximum theoretical efficiency of 100\%) and the 3D-CFD simulations, even with existing losses, turbulence effects, anisotropy in the zones of high flow circulation, and scale effects, which are not considered neither in the theoretical methodology for BMC or in CFD analyses. CFD simulation use, in the first stage, angles obtained by BMC, but a further investigation of the best-efficiency operating conditions require small corrections to these angles, as shown in Table 2.

It is also worth mentioning the difference in power values between impellers with diameters of 100 and $200 \mathrm{~mm}$ due to essentially differences in the flow values. Two geometrically similar turbines operating at rotational speeds, which satisfy the condition presented in (11), usually have different efficiency values, in particular when the relationship between the homologous lengths is high.

$$
\frac{N}{N^{\prime}}=\left(\frac{H}{H^{\prime}}\right)^{1 / 2} \frac{D^{\prime}}{D} .
$$

The difference in efficiency values is caused by scale effects, driven by the effect of viscosity, which causes loss of pressure, thus preventing a quadratic variation of the flow velocity.

In the micro-tubular propeller of $D=200 \mathrm{~mm}$, the efficiency (Figure 8) is then obtained for different rotational speed values and flow conditions. Figure 8 also shows the curves of head and mechanical power versus discharge, and Figure 9 shows the performance curves for head and efficiency versus discharge number variation for the microtubular propeller with $D=100 \mathrm{~mm}$ tested in laboratory.

4.2. Hydrodynamic Behaviour. Once the BEP was established for the micro-tubular propeller based on mathematical modelling through the BMC model and CFD simulations, a detailed analysis was developed in order to better understand the $3 \mathrm{D}$ hydrodynamic behaviour of the flow throughout the impeller, as documented by Scott-Pomerantz [21]. Corresponding flow velocities, total pressure, and wall shear stress are presented in Figure 10, for the micro-tubular propeller 


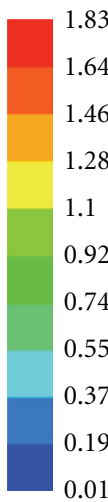

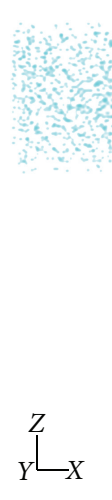

(a)

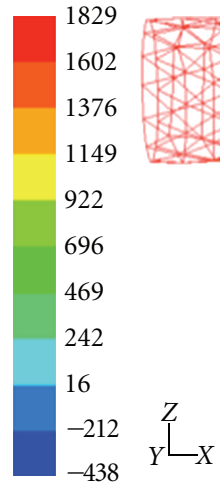

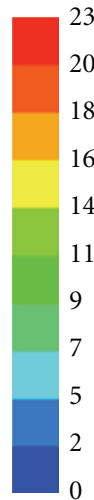

$Y^{Z}-X$

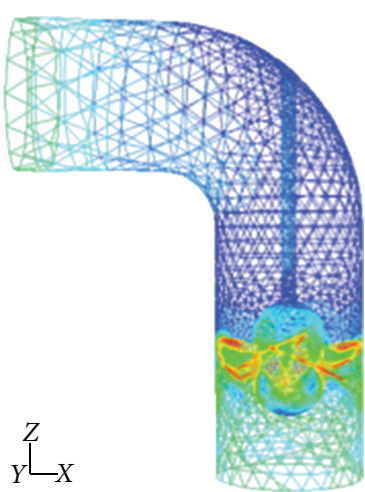

(c)

FIgURE 10: Fluid behaviour inside the micro-tubular propeller.

TABLE 2: Comparisons between BMC and CFD for the tip and hub angles.

\begin{tabular}{|c|c|c|c|c|}
\hline \multicolumn{5}{|c|}{ Methodology } \\
\hline \multirow{3}{*}{ Characteristic parameters } & \multicolumn{2}{|c|}{$\mathrm{BMC}$} & \multicolumn{2}{|c|}{ CFD } \\
\hline & $D=100 \mathrm{~mm}$ & $D=200 \mathrm{~mm}$ & $D=100 \mathrm{~mm}$ & $D=200 \mathrm{~mm}$ \\
\hline & \multicolumn{2}{|c|}{$N_{\text {sqt }}=80 \mathrm{rpm}$} & \multicolumn{2}{|c|}{$N_{\mathrm{sqt}}=77 \mathrm{rpm}$} \\
\hline Inlet hub angle $\left(\alpha_{1}\right)\left(^{\circ}\right)$ & 41 & 46 & & \\
\hline Outlet hub angle $\left(\alpha_{2}\right)\left(^{\circ}\right)$ & 17 & 11 & & \\
\hline Inlet tip angle $\left(\alpha_{1}\right)\left(^{\circ}\right)$ & 23 & 24 & & \\
\hline Outlet tip angle $\left(\alpha_{2}\right)\left(^{\circ}\right)$ & 16 & 14 & & \\
\hline Power $(\mathrm{W})$ & 4.7 & 9967 & 4.2 & 9869 \\
\hline
\end{tabular}

tested in laboratory ( $D=100 \mathrm{~mm})$. This 3D fluid-computational analysis considers steady-state pressurized flow conditions, keeping a constant rotational speed, where the singularities reflect an increase of turbulence. Analysis of the same figure shows flow disturbances inside the turbine. This is not only due to the rotation of the impeller, as it is associated to the circulation flow, but also to the way in which the flow enters into the turbine section, going through the propeller and leaving with a rotational movement (in a vortex configuration) towards the draft tube. Upstream of the turbine, the flow has a low velocity and higher-pressure values, exhibiting an irrotational behaviour. At the turbine section the flow is influenced by the impeller contour, which induces a flow separation effect which significantly affects the turbulence, as reflected by the wall shear stress. It is also noticed that the shear stress is higher near the periphery of the blades, inducing a significant flow resistance in this zone.

For this micro-tubular turbine design, four sectioning plans (Figure 11) are used to analyse the behaviour of the flow in zones where the flow range can vary and where a better understanding about its behaviour is needed. In Figure 12, the fluid enters the turbine with an average speed of $0.52 \mathrm{~m} / \mathrm{s}$, which decreases as the flow approaches the tubular walls due to the effect caused by wall friction. As it approaches the curve and the impeller, the flow exhibits a nonlinear behaviour in the velocity distribution. Along the axis, the flow tends to be influenced by the shaft rotation, which induces the formation of separation zones. The

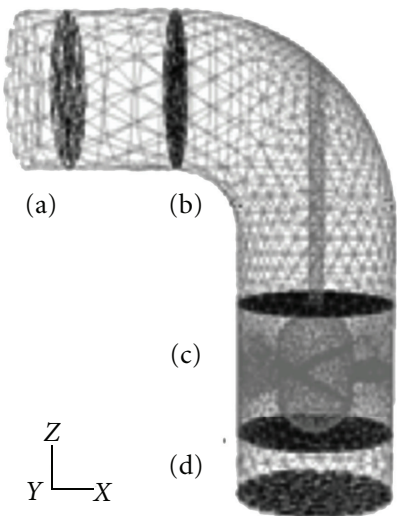

Figure 11: Schematic representations of the sectioning plans for instantaneous velocity analysis.

sectioning plans along the turbine allow the confirmation and visualization of the flow behaviour occurring through the propeller.

\section{Comparisons and Results Discussion}

Comparing Figure 7 (experiments) with Figure 12 (CFD simulations), a similar behaviour of the fluid is evident, as well as the identification of the section in which the separation effect is more significant. When the fluid comes 


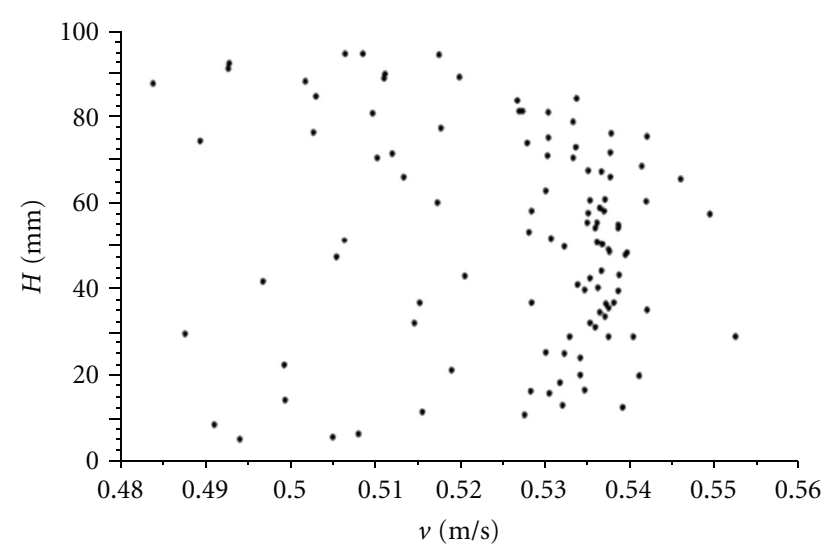

(a)

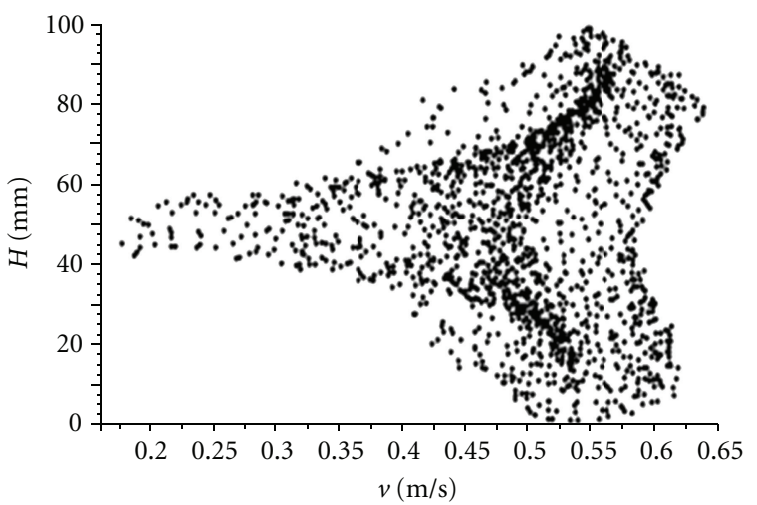

(c)

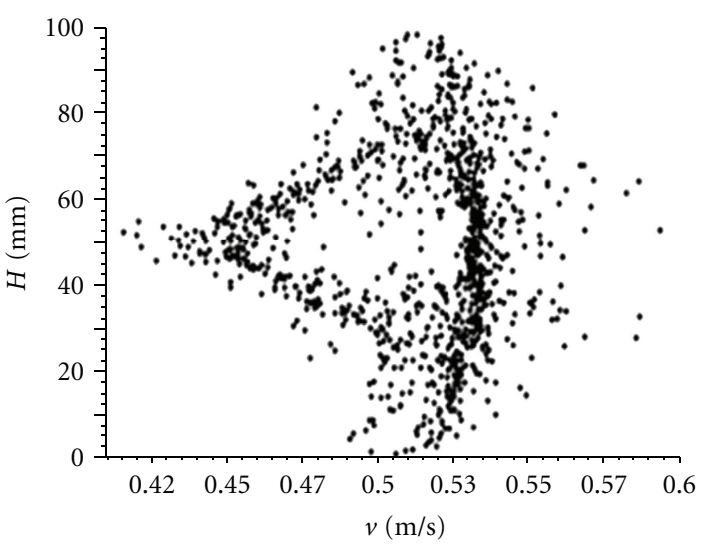

(b)

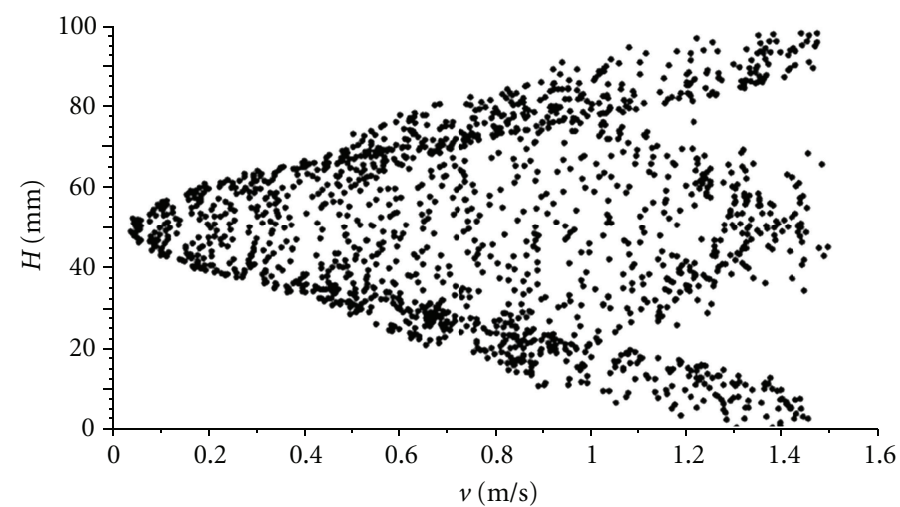

(d)

FIGURE 12: CFD simulations for the flow velocity variation across the turbine.

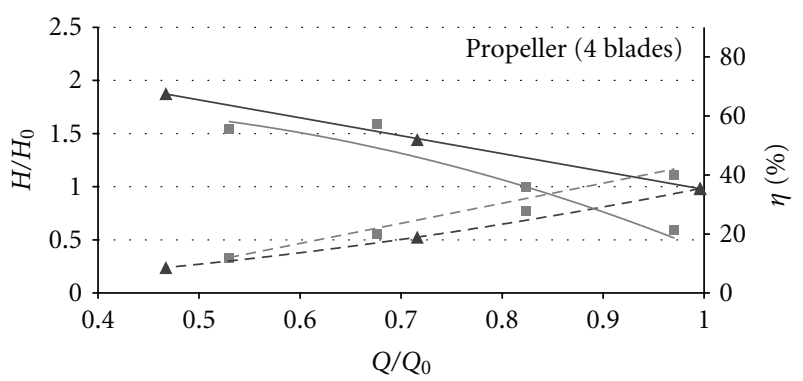

- $\operatorname{Exp}(N=70 \mathrm{rpm})$

- $\operatorname{CFD}(N=70 \mathrm{rpm})$

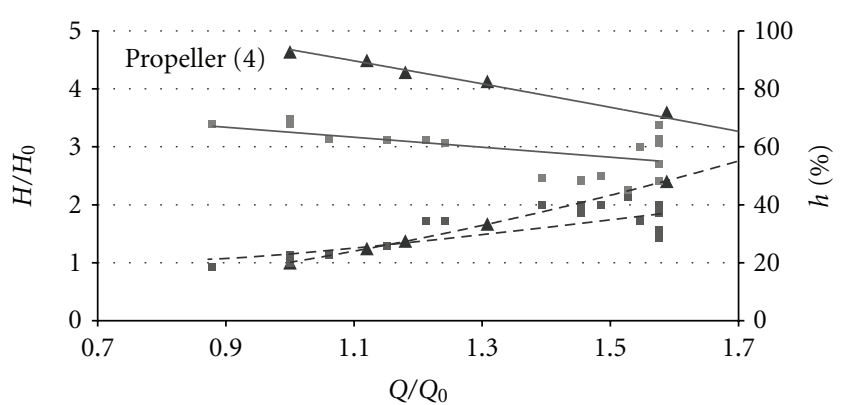

- $\operatorname{Exp}(N=200 \mathrm{rpm})$

$\triangle \operatorname{CFD}(N=200 \mathrm{rpm})$

- - $H / H_{0}$ variation

(a)

(b)

FIGURE 13: Comparison between CFD and experimental performance curves for rotational speeds of 70 and $200 \mathrm{rpm}$.

closer to the curve where the turbine shaft is located, there is certain anisotropy with velocity retardation induced by the impeller rotation. Once the flow passes through the bulb, pressure and velocity decrease, induced by the sub-pressure existing downstream of the impeller. This leads to the formation of a turbulent wake zone, as established by Ramos et al. [22]. Along the turbine the fluid moves into two regions: (1) a thin layer near the solid walls, in which the tangential stress play is an important role (the boundary layer); and (2) the remaining part is fullfilled where the shear stress is less significant.

Dimensionless characteristic parameters of CFD simulations and laboratory tests were selected and compared as shown in Figure 13. In this figure, $H_{0}$ and $Q_{0}$ are the rated 
TABLE 3: Main characteristics of the micro-tubular propeller for low-head solutions.

\begin{tabular}{lccccccccc}
\hline Turbine & $D$ & $H_{0}$ & $Q_{0}$ & $N_{0}$ & $\eta_{\max }$ & $P_{\text {mec }}$ & \multicolumn{2}{c}{ Range of application } \\
& $(\mathrm{mm})$ & $(\mathrm{m})$ & $\left(\mathrm{m}^{3} / \mathrm{s}\right)$ & $(\mathrm{rpm})$ & $(\%)$ & $(\mathrm{W})$ & $Q\left(10^{-3} \mathrm{~m}^{3} / \mathrm{s}\right)$ & $H(\mathrm{~m})$ & $N(\mathrm{rpm})$ \\
\hline \multirow{2}{*}{ Propeller with 4 blades } & $100^{(1)}$ & 0.23 & 0.005 & 300 & $65-70$ & 8 & $3-8$ & $0.05-0.30$ & $100-300$ \\
& $200^{(2)}$ & 10 & 0.109 & 1000 & $65-80$ & 10000 & $110-200$ & $5-18$ & $900-1400$ \\
\hline
\end{tabular}

${ }^{(1)} \mathrm{CFD}$ and experimental analysis for lab conditions; ${ }^{(2)} \mathrm{CFD}$ similarity analysis.

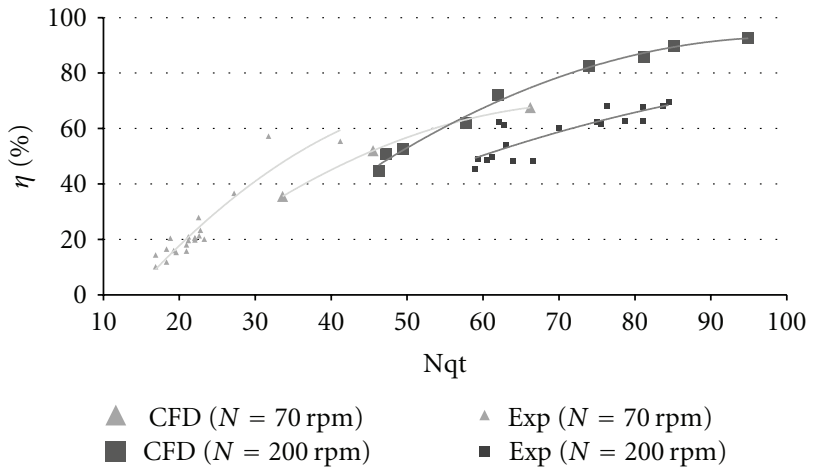

FIGURE 14: Comparison of efficiency versus specific speed values between CFD and experiments for rotational speeds of 70 and $200 \mathrm{rpm}$.

values of head and discharge, respectively. The comparison of 3D-CFD simulations with laboratory test results shows a reasonable fit of the head performance curve and a worse adjustment in the efficiencies due to scale effects, torque measure accuracy due to mechanical friction in the balance brake system and in bearings, and in leaks between the impeller and the external envelop. CFD simulations are not able to take into account these effects. Figure 14 presents a comparison between efficiency versus specific speed $\left(\mathrm{m}, \mathrm{m}^{3} / \mathrm{s}\right)$ curves for two rotational speed values (i.e., 70 and $200 \mathrm{rpm}$ ). Once again the efficiency values obtained by $3 \mathrm{D}-\mathrm{CFD}$ are higher than the experimental ones as former confirmed.

\section{Concluding Remarks}

An extended analysis based on laboratory testing and numerical modelling in a new prototype micro-propeller design is presented. A summary of the main characteristics of the converter developed in this study at two scales $(D=100 \mathrm{~mm}$ limited to small discharges - and $D=200 \mathrm{~mm}$-adequate for higher discharges and power) is shown in Table 3. This type of machine is usually composed by a runner installed in a pipe curve, without volute or guide vane as simple as possible to be a cost-effective solution and easy to be implemented in a bypass into existing water infrastructures. They are appropriate for operating under almost constant-flow conditions, such as in water supply systems equipped with discharge control valves or tanks with capacity to regulate. The use of the blade model configuration (BMC), together with experimental tests and 3D fluid computational analyses, can help researchers and equipment manufacturers to better understand the phenomenon associated with the hydrodynamic and a turbine design. This can lead to a greater knowledge on the interaction between the machine geometry, the hydraulic flow conditions, and the turbine performance.

This paper highlights the importance of using extended testing and computational analysis which to guide the design of new energy solutions for micro-hydro schemes. These solutions come as a promising answer to cover the lack of energy in isolated or rural zones, or in pressurized systems for water transport.

\section{Nomenclature}

c: Periphery velocity $\left(\mathrm{m} \mathrm{s}^{-1}\right)$. Subscripts 1 for inlet and subscript 2 for outlet

$D$ : Pipe diameter or outside diameter of the blades $(\mathrm{m})$

$g$ : Gravity acceleration $\left(\mathrm{m} / \mathrm{s}^{2}\right)$

$H$ : Net head (m)

$K$ : Constant of the free vortex $(-)$

$M$ : Torque ( $\mathrm{N} \mathrm{m}$ )

$N$ : Rotational speed (rpm). Subscript sqt for Specific rotational speed

$P$ : Power (W). Subscript $h$ for hydraulic power and subscript mec for mechanical power

Q: Discharge $\left(\mathrm{m}^{3} \mathrm{~s}^{-1}\right)$

$r$ : Blade radius $(\mathrm{m})$. Subscript $e$ for tip and subscript $i$ for hub.

S: Tubular cross-section area $\left(\mathrm{m}^{2}\right)$

$v$ : Flow velocity $\left(\mathrm{m} \mathrm{s}^{-1}\right)$. Subscript 1 and 2 for the inlet and outlet of the blade

\section{Greek Symbols}

$\alpha$ : Blade angle on the periphery $\left(^{\circ}\right)$. Subscripts 1 for inlet and subscript 2 for outlet

$\gamma$ : Specific weight of the fluid $\left(\mathrm{N} \mathrm{m}^{-3}\right)$

$\eta_{t}$ : Efficiency $(-)$

$\rho$ : Fluid mass density $\left(\mathrm{kg} \mathrm{m}^{-3}\right)$

$\varphi$ : Discharge number $(-)$

$\Psi$ : Head number $(-)$

$\omega$ : Impeller rotational speed $\left(\mathrm{rad} \mathrm{s}^{-1}\right)$.

\section{Acknowledgments}

The authors are grateful to the EU HYLOW project from the EU 7th Framework Programme (Grant no. 212423) and to the Portuguese FCT-funded project (PTDC/ECM/65731/ 2006) for the contribution to the development of laboratory 
and computational dynamic research work associated with the performance of a new prototype micro-tubular propeller turbine.

\section{References}

[1] R. J. Blakely and K. F. O'Connor, "Present and potential use of micro-hydro-electric schemes in remote locations," Tech. Rep., New Zealand Energy Research and Development Committee, Auckland, New Zealand, 1981.

[2] R. J. Campbell, Small Hydro and Low-Head Hydro Power Technologies and Prospects, Congressional Research Center, March 2010.

[3] D. A. Howey, "Axial flux permanent magnet generators for pico-hydropower," in Proceedings of the Engineers Without Borders UK Research Conference, The Royal Academy of Engineering, London, UK, February 2009.

[4] R. G. Simpson and A. A. Williams, "Application of computational fluid dynamics to the design of pico propeller turbines," in Proceedings of the International Conference on Renewable Energy for Developing Countries (ICREDC '06), School of Engineering and Applied Sciences, University of the District of Columbia, Washington, DC, USA, 2006.

[5] G. M. Demetriades, Design of low-cost propeller turbines for standalone micro-hydroelectric generation units, Ph.D. dissertation, University of Nottingham, Nottingham, UK, 1997.

[6] D. Upadhyay, Low head turbine development using computational fluid dynamics, Ph.D. dissertation, University of Nottingham, Nottingham, UK, 2004.

[7] K. V. Alexander, E. P. Giddens, and A. M. Fuller, "Axial-flow turbines for low head microhydro systems," Renewable Energy, vol. 34, no. 1, pp. 35-47, 2009.

[8] K. V. Alexander, A. M. Fuller, and E. P. Giddens, "Radial- and mixed-flow turbines for low head microhydro systems," Renewable Energy, vol. 34, no. 7, pp. 1885-1894, 2009.

[9] K. V. Alexander and E. P. Giddens, "Microhydro: cost-effective, modular systems for low heads," Renewable Energy, vol. 33, no. 6, pp. 1379-1391, 2008.

[10] K. V. Alexander and E. P. Giddens, "Optimum penstocks for low head microhydro schemes," Renewable Energy, vol. 33, no. 3, pp. 507-519, 2008.

[11] A. Skotak, J. Mikulasek, and J. Obrovsky, "Development of the new high specific speed fixed blade turbine runner," The International Journal of Fluid Machinery and Systems, vol. 2, no. $4,2009$.

[12] H. Olgun, "Investigation of the performance of a cross-flow turbine," International Journal of Energy Research, vol. 22, no. 11, pp. 953-964, 1998.

[13] H. Olgun, "Effect of interior guide tubes in cross-flow turbine runner on turbine performance," International Journal of Energy Research, vol. 24, no. 11, pp. 953-964, 2000.

[14] K. Sumathy, A. Venkatesh, and V. Sriramulu, "Effect of discharge head on the performance of a solar water pump," International Journal of Energy Research, vol. 18, no. 6, pp. 623-629, 1994.

[15] P. R. Krishnamoorthy, S. Seetharamu, and M. S. Bhatt, "Design optimization of a biomass-fuelled steam-operated pistonless pump," International Journal of Energy Research, vol. 15, no. 5, pp. 347-356, 1991.

[16] P. Sutikno and I. K. Adam, "Design, simulation and experimental of the very low head turbine with minimum pressure and free vortex criterions," International Journal of Mechanical \& Mechatronics Engineering, vol. 11, no. 1, 2011.
[17] M. Kaniecki and Z. Krzemianowski, "Recent experience of IFFM PAS in the design process of lowhead propeller hydraulic turbines for Small Hydro," IOP Conference Series: Earth and Environmental Science, vol. 12, no. 1, Article ID 012069, 2010.

[18] H. M. Ramos, A. Borga, and M. Simão, "Cost-effective energy production in water pipe systems: theoretical analysis for new design solutions," in Proceedings of the 33rd IAHR Congress. Water Engineering for a Sustainable Environment, Managed by EWRI of ASCE on behalf of IAHR, British Columbia, Canada, August 2009.

[19] S. Rawal and J. T. Kshirsagar, "Numerical simulation on a pump operating in a turbine mode," in Proceedings of the 23rd International Pump Users Symposium, pp. 21-27, Texas A\&M University, Houston, Tex, USA, 2007.

[20] H. M. Ramos, A. Borga, and M. Simão, "New design solutions for low-power energy production in water pipe systems," Water Science and Engineering, vol. 2, no. 4, pp. 69-84, 2009.

[21] C. D. Scott-Pomerantz, The k-epsilon model in the theory of turbulence, Ph.D. dissertation, University of Pittsburgh, Pittsburgh, Pa, USA, 2004.

[22] H. M. Ramos, A. Borga, and M. Simão, "Energy production in water supply systems: computational analysis for new design solutions," in Environmental Hydraulics, P. A. López-Jiménez et al., Ed., Taylor \& Francis, London, UK, 2010. 

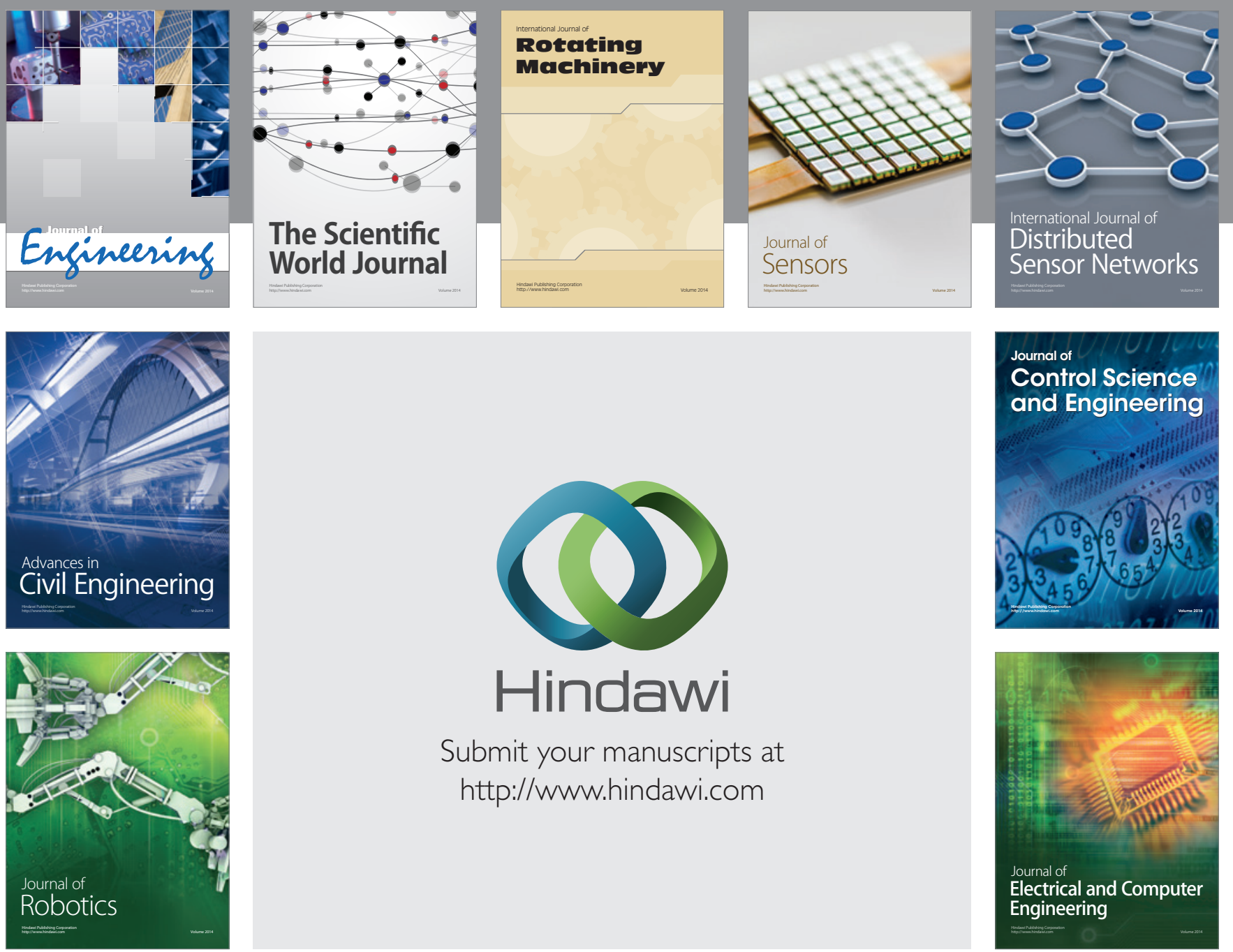

Submit your manuscripts at

http://www.hindawi.com
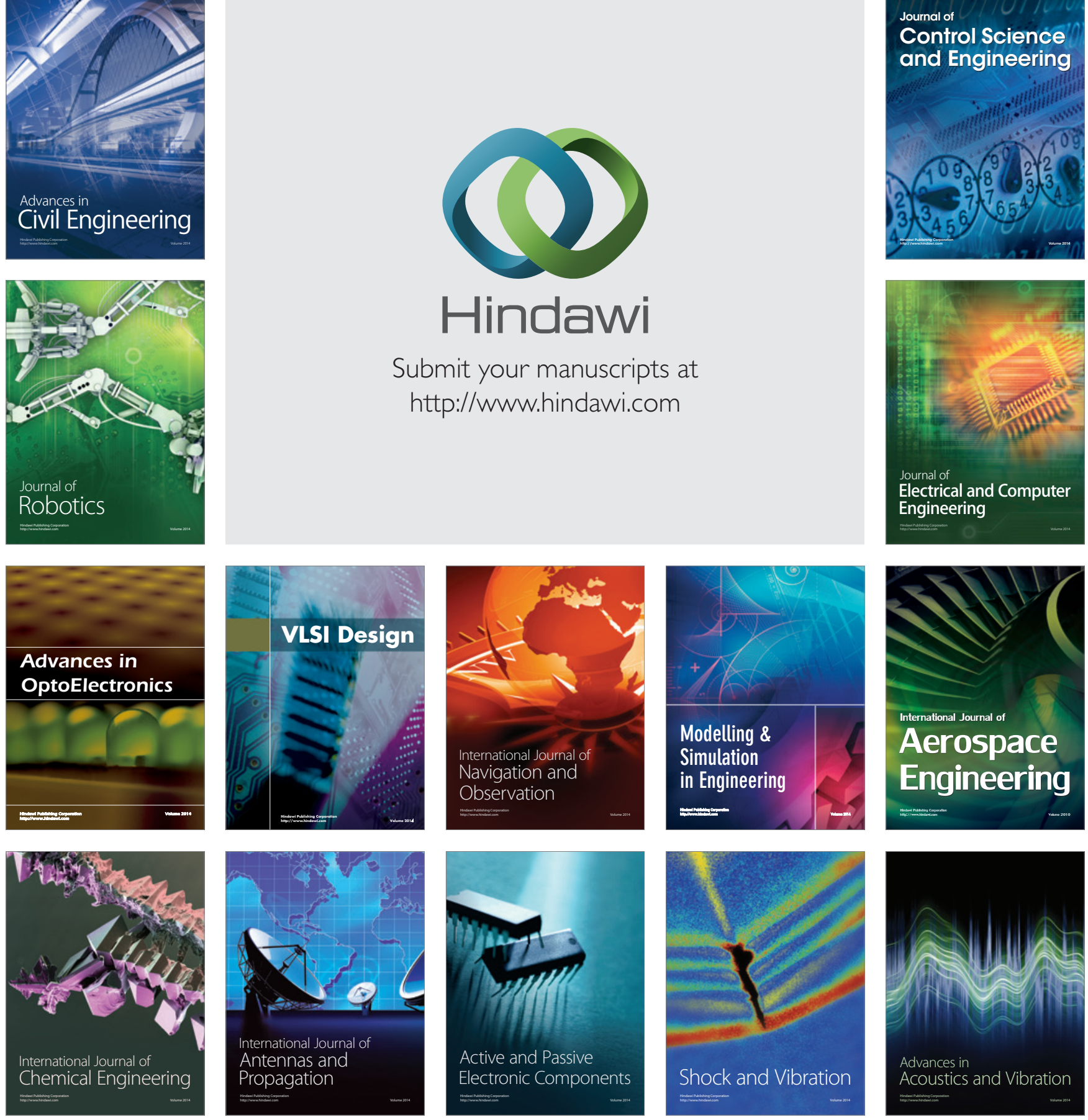